\title{
Ensino remoto na pandemia de COVID-19: lições aprendidas em um projeto de extensão universitário
}

\author{
Remote teaching during the COVID-19 pandemic: lessons learned from university \\ extension project
}

Tárcila Lorrane Fernandes de Souza Soares Estudante do Bacharelado Interdisciplinar em Saúde Universidade Federal do Sul da Bahia - UFSB. Itabuna, Bahia - Brasil. tarcilafernandes97@gmail.com

Ícaro Silva de Santana Estudante do Bacharelado Interdisciplinar em Saúde Universidade Federal do Sul da Bahia - UFSB. Itabuna, Bahia - Brasil. santan.icaro@hotmail.com

Maria Luiza Caires Comper Doutora em Fisioterapia Universidade Federal do Sul da Bahia - UFSB. Itabuna, Bahia - Brasil. maria.luiza@ufsb.edu.br

Resumo: O distanciamento social, decorrente da pandemia da COVID-19, resultou na suspensão das aulas presenciais em todo mundo, fazendo com que os métodos de educação fossem rapidamente adaptados para serem ministrados no formato de ensino remoto de emergência. Essa adaptação inesperada impactou estudantes e professores que, juntos, empreenderam esforços para a experimentação de novas formas de ensinar e aprender a fim de manter a continuidade das atividades de ensino, pesquisa e extensão. É neste contexto, que este artigo se propõe a descrever o processo de adaptação das atividades de ensino de um projeto de extensão universitário para a modalidade de ensino remoto, compartilhando experiências e lições aprendidas que podem contribuir para o desenho de novas estratégias pedagógicas.

Palavras-chave: COVID-19. Ensino remoto. Tecnologias Digitais. Metodologias Ativas.

Abstract: The social distance caused by the COVID-19 pandemic has resulted in the suspension of faceto-face classes around the world. Educational methods needed to be quickly adapted to the remote learning. This unexpected adaptation impacted students and teachers who, together, committed themselves to experimenting new forms of teaching and learning in order to maintain the continuity of teaching, research and extension activities. Therefore, the goal of this paper is to describe the process of adapting a university extension project to the remote learning. It is also proposed to share experiences and learnings that can contribute to the design of new pedagogical strategies that provide the student's autonomy in the learning process in this new pandemic reality.

Key-Word: COVID-19. Remote Learning. Digital Technologies. Active teaching methodologies. 
SOARES, Tárcila Lorrane Fernandes de Souza; SANTANA; Ícaro Silva de; COMPER, Maria Luiza Caires. Ensino remoto na pandemia de COVID-19: lições aprendidas em um projeto de extensão universitário

\section{Introdução}

O SARS-COV-2, vírus causador da COVID-19, tem causado grandes preocupações e repercussões em todo o mundo. Trata-se de doença respiratória com sintomas e gravidade variável que foi notificada pela primeira vez em dezembro de 2019, na cidade de Wuhan, China. Inicialmente, acreditava-se que este seria um problema local. No entanto, o aumento exponencial do número de casos fez com que a Organização Mundial de Saúde (OMS) declarasse a situação como uma pandemia (WHO, 2020). Desde então, o distanciamento social tem sido recomendado como a estratégia mais eficiente para o enfrentamento do novo vírus (COUTO et al, 2020).

O distanciamento social, dentre outras coisas, resultou na suspensão das aulas presenciais na educação básica e no ensino superior, fazendo com que os métodos de educação fossem rapidamente adaptados para serem ministrados no formato de ensino remoto de emergência (SANDHU; DE WOLF, 2020). No Brasil, o Ministério da Educação (MEC) aprovou a Portaria no 343 de 17 de março de 2020 que dispõe sobre a substituição das aulas presenciais por aulas em meios digitais enquanto durar a situação de pandemia do Novo Coronavírus - COVID-19 (BRASIL, 2020).

O ensino remoto de emergência é uma modalidade alternativa que busca soluções de ensino por meio de tecnologias digitais para manutenção da rotina de aulas que seriam ministradas presencialmente (HODGES et al., 2020). O objetivo é disponibilizar conteúdos educacionais possíveis de serem acessados de qualquer lugar geográfico por um aparelho conectado à internet (TAKAHASHI; CARDOSO, 2012). A grande questão é que a mudança na modalidade de ensino, especialmente de modo tão abrupto, não é tão simples como parece. Ao contrário, ela impacta estudantes e professores que, juntos, precisam empreender esforços para encontrar as melhores estratégias de ensino e aprendizagem que atendam às novas necessidades impostas pelo distanciamento social (AQUINO et al, 2020).

Diante desse cenário, o projeto de extensão da Universidade Federal do Sul da Bahia (UFSB), denominado de Núcleo de Estudos em Semiologia e Propedêutica clínica (NESPc), viuse na condição de adaptar suas atividades, outrora presenciais, para a modalidade de ensino remoto. O NESPc caracteriza-se como um projeto de educação continuada interprofissional, que visa complementar, aprofundar, atualizar e difundir conhecimentos da semiologia e propedêutica clínica, da epidemiologia preventiva e da educação em saúde. Suas atividades estão organizadas em quatro eixos, dos quais se destacam as atividades de ensino realizadas semanalmente (ALMEIDA; DE SANTANA; COMPER, 2019). 
Este artigo se propõe a descrever o processo de adaptação das atividades de ensino de um projeto de extensão universitário para a modalidade de ensino remoto de emergência, compartilhando experiências e lições aprendidas que podem contribuir para o desenho de novas estratégias pedagógicas a serem utilizadas no ensino remoto.

\section{Métodos}

Trata-se de um relato de experiência que, ao adaptar as atividades de para o ensino remoto, buscou ressignificar as práticas pedagógicas e os espaços de compartilhamento de saberes e práticas. Para tanto, utilizou-se como método a descrição do planejamento e organização e realização dos ciclos de estudo, considerando os principais resultados alcançados. Tais resultados emergiram de observações, relatos e discussões entre docentes e estudantes durante a realização da adaptação do projeto.

O NESPc é um projeto de extensão criado e desenvolvido na UFSB - Campus Jorge Amado e vinculado ao curso de Bacharelado Interdisciplinar em Saúde (BIS). É caracterizado como um espaço de discussão e produção de conhecimentos relacionados à propedêutica clínica, epidemiologia preventiva e educação em saúde. O NESPc tem por objetivo principal a educação continuada de estudantes e profissionais de diferentes cursos de graduação da área da saúde. Em prol desses objetivos, o NESPc se alicerça em atividades de ensino, pesquisa e extensão e contribui para incorporação de saberes e práticas numa perspectiva de educação interprofissional condizentes com a proposta de formação do BIS (ALMEIDA; DE SANTANA; COMPER, 2019).

Atualmente, o NESPc possui 25 participantes que são organizados em quatro Equipes de Aprendizagem Ativa (EAA). Os participantes são estudantes, profissionais e docentes do BIS e de cursos de graduação em biomedicina, enfermagem, fisioterapia, medicina, odontologia e psicologia de diferentes instituições de ensino da região. Cada EAA é responsável por um dos 4 eixos temáticos de condução do ciclo de estudos: Bases Morfofuncionais (BMF), Semiologia e Propedêutica Clínica Aplicada (SPCA), Prática Baseada em Evidências (PBE) e Promoção da Saúde e Qualidade de Vida (PSQV).

As atividades de ensino seguem um planejamento mensal, orientado por uma pergunta geradora relacionada às condições de saúde dos diferentes ciclos de vida. A cada semana, uma EAA é responsável por planejar e conduzir a sessão de estudos. Um detalhamento maior desse projeto de extensão e de seus resultados pode ser encontrado no estudo de Almeida; Santana; Comper, 2019 (ALMEIDA; DE SANTANA; COMPER, 2019). 
Os resultados obtidos no processo de adaptação do projeto de extensão para a modalidade de ensino remoto foram analisados considerando o contexto (necessidades, problemas, ferramentas e oportunidades), a entrada (estratégia, plano de ação, organização da equipe e o orçamento), os elementos do processo (implementação de estratégias e planos) e o produto (aprendizado) (HODGES et al., 2020).

\section{Planejamento dos ciclos de estudo}

As atividades do NESPc foram interrompidas em meados de março em razão das orientações para o distanciamento social. Sem a perspectiva de um retorno imediato, os participantes sugeriram que os encontros presenciais fossem adaptados para um modelo de ensino remoto apoiado em tecnologias de informação e comunicação (TICS). Assim, as sessões de ensino foram organizadas em dois ciclos de estudos a partir do tema gerador: COVID-19 e suas repercussões na saúde das pessoas nos diferentes ciclos de vida.

O primeiro ciclo, denominado “Questões clínicas e epidemiológicas da COVID-19” teve como objetivo discutir os elementos conceituais, clínicos, epidemiológicos e de evidências científicas relacionados à COVID-19. O segundo ciclo foi denominado de "Repercussões do distanciamento social nos ciclos de vida" e teve o objetivo de aprofundar o conhecimento e promover reflexões relacionadas às repercussões do distanciamento social na saúde física e mental dos indivíduos em seus diferentes ciclos de vida. Essa organização foi proposta com o objetivo de discutir o cuidado em saúde durante e após a pandemia por meio de uma perspectiva mais abrangente, colaborativa e interprofissional.

Um cronograma com os temas, datas e equipes responsáveis pela condução das sessões de ensino foi elaborado e compartilhado com os participantes, com vistas à auxiliar no planejamento e execução das tarefas. Materiais de divulgação para convite do público externo foram confeccionados semanalmente para transmissão via mídias digitais e Instagram do NESPc (@nespc.ufsb).

Os encontros ocorreram semanalmente, às quintas-feiras, das 16 às 18 horas, no período de 30 de abril ao dia 18 de junho de 2020. Os temas trabalhados seguiram a sequência apresentada no fluxograma da Imagem 1, conforme o planejamento realizado. Cada EAA foi responsável por conduzir e organizar uma sessão de ensino dos temas apresentados no fluxograma. 
SOARES, Tárcila Lorrane Fernandes de Souza; SANTANA; Ícaro Silva de; COMPER, Maria Luiza Caires. Ensino remoto na pandemia de COVID-19: lições aprendidas em um projeto de extensão universitário

Imagem 1 - Fluxograma das sessões de ensino remoto do NESPc para o ciclo de estudos: COVID19 e suas repercussões na saúde das pessoas nos diferentes ciclos de vida

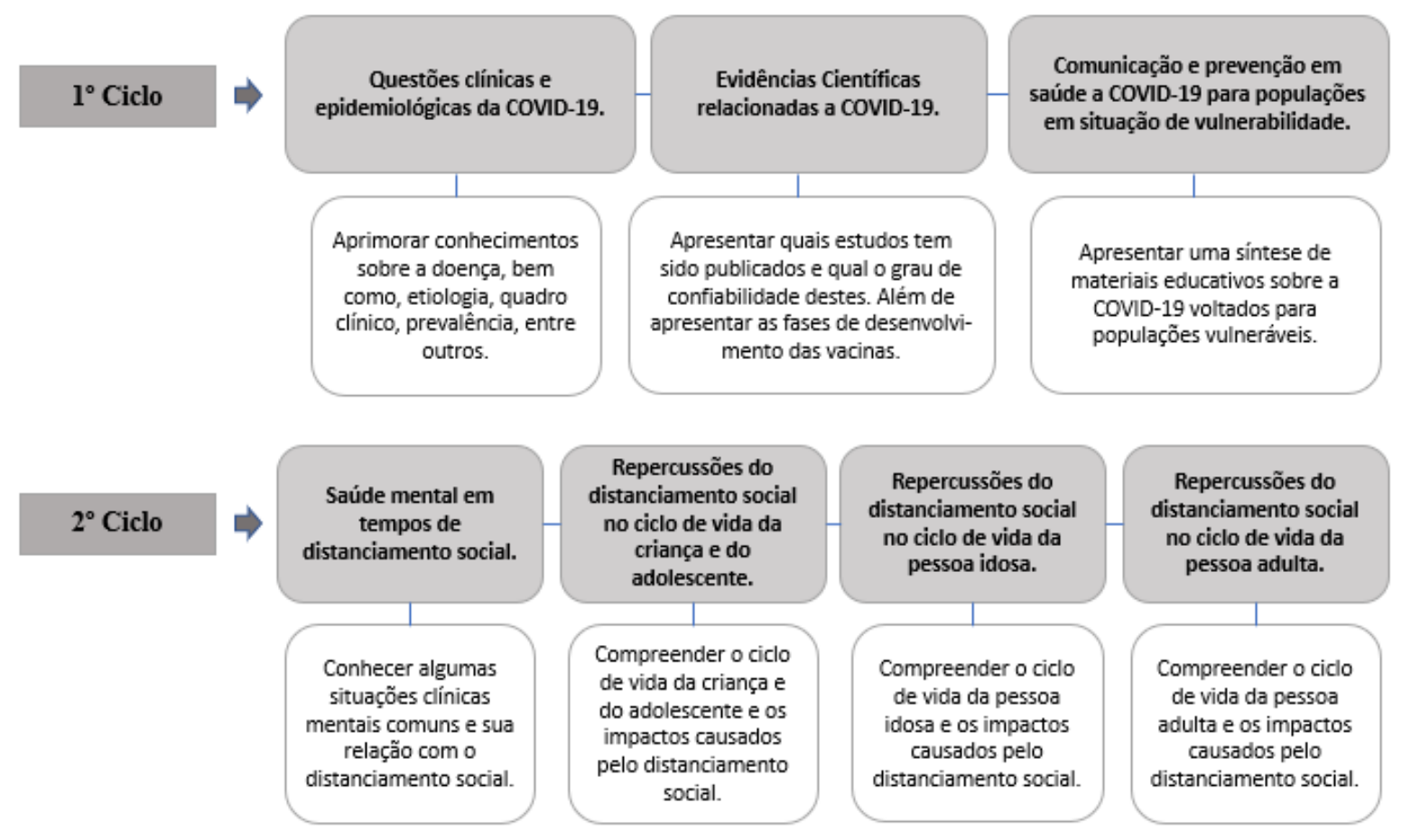

Fonte: Própria dos autores, 2020.

Cada sessão de ensino foi planejada pelos membros da EAA responsável, com orientação docente. Para tanto, foram consideradas as perguntas norteadoras relacionadas ao tema da semana, as estratégias pedagógicas e materiais (referências bibliográficas, audiovisuais e outros) a serem utilizados e o palestrante convidado. A estratégia pedagógica predominante foi a exposição dialogada. Os materiais utilizados nas apresentações foram produzidos a partir de buscas em bases de dados, livros ou sites. Os palestrantes convidados foram selecionados de acordo a sua expertise no assunto abordado na semana e convidados pela professora coordenadora ou pela equipe responsável.

Algumas ferramentas digitais foram utilizadas para o planejamento e realização das sessões de ensino. O aplicativo WhatsApp foi fundamental no estabelecimento de diálogos com os membros da equipe por permitir o envio de mensagens de texto, arquivos, áudios, vídeos etc. Além disso, foram utilizadas as ferramentas Google Meet, documentos Google e apresentações Google para, respectivamente, realizar conferências a fim de discutir o tema e dividir tarefas entre os membros; elaborar o roteiro da sessão de ensino; e, confeccionar as apresentações em slides. Para a transmissão das sessões de ensino, utilizou-se a plataforma Microsoft Teams, sendo o link 
previamente enviado para os integrantes do NESPc e disponibilizado nas redes sociais para convite de participantes externos.

\section{Resultados alcançados}

Em termos gerais, é possível afirmar que houve viabilidade para adaptação do projeto de extensão do NESPc para o modelo de ensino remoto de emergência. Acreditamos que uma das principais razões para este resultado tenha sido o engajamento de estudantes e docentes em retomar as atividades do projeto como alternativa de redução dos prejuízos no processo de ensino e aprendizagem (ALVES, 2020). As plataformas digitais gratuitas também favoreceram a adaptação por possibilitar a interação entre os participantes de diversas localizações geográficas (CARVALHO; CONCEIÇÃO; GOIS, 2020).

A avaliação do planejamento dos ciclos de estudo para o ensino remoto mostrou pontos positivos e negativos que merecem atenção. Um dos pontos positivos foi a organização de conteúdos trabalhados nos ciclos de estudos. Isso permitiu que os participantes tivessem, inicialmente, uma visão ampliada das questões epidemiológicas e clínicas da COVID-19 e, depois, de como seriam as repercussões do distanciamento social na saúde das pessoas nos diferentes ciclos de vida.

Outro ponto positivo foi o desenvolvimento de novas habilidades para manejo das ferramentas digitais, utilizadas para o planejamento e realização das sessões de ensino. Observouse uma evolução no que diz respeito ao domínio para seleção e manuseio de cada ferramenta, de acordo com o objetivo pedagógico proposto para a sessão de ensino.

A participação de professores convidados para condução das exposições dialogadas mostrou resultados conflituosos. Isso porque, a participação de um expert oportunizou uma troca de conhecimentos atualizados, embasados cientificamente e exemplificados para o contexto real do cuidado em saúde. Porém, a estratégia de exposição dialogada não favoreceu a interação entre os participantes ouvintes. A ausência de um ambiente virtual para compartilhamento do planejamento das sessões de ensino e de materiais para estudos prévios também pode ter colaborado com este resultado.

A baixa interação entre participantes na sala virtual foi um ponto negativo de destaque, especialmente quando comparado ao ensino presencial. A maior parte dos participantes mantiveram suas câmeras e microfones desligados e os questionamentos, as dúvidas e os posicionamentos foram menos frequentes. Tais fatores podem estar relacionados à queda ou 
ausência de conexão para acessar a plataforma digital, receio da exposição em um ambiente online, dificuldade em se adaptar a nova metodologia de ensino, entre outros.

O produto final resultante do ciclo de estudos foram: 06 sessões de ensino, 6 resumos científicos e elaboração do artigo em tela. Cada sessão obteve, em média, a presença de 40-50 participantes que incluíram estudantes da UFSB e de outras instituições, profissionais de saúde e outros ouvintes da comunidade. As produções foram submetidas e aprovadas em congressos científicos virtuais, realizados durante a pandemia.

Os participantes indicaram ao final do ciclo de estudos que as questões discutidas contribuíram para ampliar seus conhecimentos sobre a COVID-19, principalmente por que o ciclo de estudos abordou elementos epidemiológicos, clínicos e psicossociais relacionados aos ciclos de vida da população impactada pela pandemia. Outro ponto muito mencionado foi a inclusão de reflexões sobre a qualidade e confiabilidade das evidências científicas que estão sendo amplamente divulgadas neste cenário de pandemia.

\section{Lições aprendidas e novas possibilidades}

Os resultados descritos anteriormente nos permitem concluir que a adaptação de um projeto de extensão universitário para a modalidade de ensino remoto foi viável e contribuiu para a formação dos estudantes. Mais que isso, a experiência vivenciada deixou algumas lições que podem contribuir para o desenho de novas estratégias pedagógicas a serem utilizadas no ensino remoto.

\section{Primeira Lição:}

Planejamento compartilhado do plano de aula

A primeira lição aprendida pauta-se na importância do planejamento do plano de aula e seu compartilhamento com os estudantes. O planejamento é um instrumento que subsidia a prática pedagógica, permitindo uma organização metodológica do conteúdo e das estratégias metodológicas a serem utilizadas para alcançar os objetivos propostos para o processo de ensino e de aprendizagem. O plano de aula é um dos produtos desse planejamento, elaborado a partir de reflexões que, em síntese, decorrem das questões: "O que pretendo com minha aula? Quais conbecimentos e/ ou habilidades eu pretendo que os estudantes desenvolvam? Qual a melhor estratégia para que isso aconteça?".

A nossa experiência mostrou que o compartilhamento do plano de trabalho traz grandes contribuições para autonomia do estudante e, consequente, êxito no processo de ensino e 
aprendizagem. Isto porque, o plano de trabalho assume a função de "guia" pedagógico, contendo as informações necessárias para que os estudantes possam mobilizar conhecimentos prévios e pesquisar/explorar informações relacionadas aos novos conhecimentos a serem discutidos (VASCONCELLOS, 2010).

Neste caso, é recomendado que o plano de aula inclua informações sobre: a) objetivos pedagógicos; b) conteúdos a serem aprendidos; c) estratégias pedagógicas a serem utilizadas; d) as atividades/produtos a serem realizados; e) referências bibliográficas, audiovisuais e de outros conteúdos digitais a serem utilizados como apoio aos estudos (MOREIRA; HENRIQUES; BARROS, 2020). É importante que este plano de trabalho seja disponibilizado no ambiente virtual para que o estudante possa acessá-lo a qualquer momento de sua rotina de estudos, seja antes, durante ou depois da aula.

\section{Segunda Lição:}

O uso do ambiente virtual de aprendizagem como apoio ao ensino remoto

A segunda lição relaciona-se com a necessidade de ter um Ambiente Virtual de Aprendizagem (AVA) que funcione como uma extensão da sala de aula e que possibilite aos estudantes, geograficamente dispersos, uma interação que pode acontecer em tempos e espaços variados. Neste sentido, o AVA deve ir além de um "espaço" para repositório de materiais, constituindo uma ferramenta de apoio ao processo de ensino e aprendizagem para: a) comunicação para planos de aula, notícias e avisos; b) armazenamento, distribuição e gerenciamento dos conteúdos de aprendizado; c) interações pedagógicas com os estudantes, com produção de conteúdos e canais variados de comunicação através de ferramentas síncronas e assíncronas; d) ferramentas de controle e avaliação do processo didático (SALVADOR et al., 2017).

Em termos de configuração, o AVA deve ser compatível com o plano de trabalho da disciplina, de modo que os conteúdos e as atividades possam ser organizados por semanas, tópicos ou perguntas geradoras. É importante que os conteúdos sejam adequados ao contexto de cada disciplina e que as diferentes ferramentas disponíveis no ambiente virtual de aprendizagem sejam utilizadas para atividades síncronas e assíncronas (LACERDA; SILVA, 2015). O AVA também favorece a adoção de metodologias ativas, pois oportuniza processos de mediação, colaboração e interatividade focados na aprendizagem do aluno.

Atualmente, existem muitas opções de ambientes virtuais de aprendizagem gratuitos, como por exemplo, Moodle (Object-Oriented Dynamic Learning Environment), AulaNet, Teleduc, E-proinfo, Eureka, Virtus (PAIVA, 2010). Interfaces web 2.0 que incluem blogs, facebook, youtube, podcasts e 
videocast também podem ser boas opções para o ensino remoto (LEÃO, 2015). Neste caso, a decisão sobre qual ambiente virtual ou de interface utilizar deve considerar, de um lado, a intencionalidade de ensino, o objetivo pedagógico e características, necessidades e/ou interesses dos participantes, e de outro, o conhecimento e conforto do professor para manejo destes (PRADO, 2001).

\section{Terceira Lição:}

Uso de metodologias ativas como estratégia pedagógica

A terceira lição está fundamentada nas idéias de Paulo Freire, especialmente quando afirma que o conhecimento só pode ser desenvolvido quando o indivíduo possui desejo de aprender e estímulo para a produção de seu próprio conhecimento (FREIRE, 1996). Em outras palavras, a estratégia pedagógica a ser utilizada no ensino remoto deve ir além do modelo formativo centrado no professor e na transmissão de conteúdos. É preciso incorporar metodologias que favoreçam processos de ensino e aprendizagem de caráter dialético, colaborativo e construtivista (LACERDA; SANTOS, 2018).

A metodologia ativa de aprendizagem é uma das alternativas a ser considerada no ensino remoto. Está concebida no modelo de educação crítico-reflexiva, valorizadora da autonomia do estudante na busca do conhecimento, por meio de pesquisa, reflexão e análise de situações/problemas (BERBEL, 2011). Neste caso, o estudante assume o protagonismo enquanto o professor passa a ser um mediador do processo de aprendizagem.

Há uma grande diversidade de metodologias ativas de ensino-aprendizagem válidas e eficientes que podem ser utilizadas nos diferentes níveis educacionais, a exemplo da aprendizagem baseada em problemas ou projetos, aprendizagem baseada em equipe, sala de aula invertida e outras (PAIVA et al., 2016). Cada metodologia possui se próprio pressuposto teórico e metodológico, que embora distinto, contribui para o desenvolvimento da autonomia do aluno; exercício do trabalho em equipe; integração entre teoria e prática; desenvolvimento de visão crítica da realidade; e, uso de avaliação formativa (BERBEL, 2011). Por isso, cabe ao professor selecionar ou mesmo criar uma nova estratégia que atenda o objetivo pedagógico proposto para a formação dos estudantes. 
SOARES, Tárcila Lorrane Fernandes de Souza; SANTANA; Ícaro Silva de; COMPER, Maria Luiza Caires. Ensino remoto na pandemia de COVID-19: lições aprendidas em um projeto de extensão universitário

\section{Quarta Lição:}

Gerenciamento da participação dos estudantes

A quarta lição aprendida diz respeito ao gerenciamento da participação dos estudantes no tocante ao compartilhamento, discussão, reflexão e construção colaborativa de conhecimentos. Esta participação pode ser motivada por todas as estratégias mencionadas anteriormente. A questão que nos apropriamos agora é: como motivar a participação e interação dos estudantes e fornecer feedbacks no ensino remoto? A resposta para tal questionamento é bastante complexa, pois envolve uma diversidade elementos tecnológicos, pessoais e sociais.

Em nossa experiência percebemos que o uso das telas abertas (vídeos) e do chat para troca de mensagens são algumas das estratégias possíveis para aumentar a interação e reduzir a dispersão dos estudantes. $\mathrm{O}$ uso do chat permite que os estudantes exponham suas dúvidas, reflexões e compartilhamento de informações em tempo real sem que exista uma interrupção no fluxo da apresentação (MARKEL; GUO, 2020). No entanto, destacamos a importância da mediação do chat pelo professor para que os comentários e questionamentos favoreçam a interação com os estudantes.

\section{Considerações finais}

É inegável que a pandemia da COVID-19 tem promovido muitas reflexões em diferentes campos de conhecimento, inclusive na educação. Isso acontece, principalmente, no que se refere à importância do ensino presencial, muitas vezes identificado como essencial para se estabelecer um aprendizado efetivo. No entanto, em um cenário onde esse modelo de ensino é inviabilizado e a tecnologia mostra-se cada vez mais presente na rotina dos indivíduos, fazer uso dela para dar continuidade à comunicação profissional, acadêmica ou familiar, tem sido um meio remediador do problema (BARBOSA; VIEGAS; BATISTA, 2020).

O processo de adaptação das atividades de ensino de um projeto de extensão universitário para a modalidade de ensino remoto apresentou resultados satisfatórios e complementares à formação dos participantes à medida que os estudantes utilizaram a inovação e criatividade. Os conhecimentos adquiridos nos ciclos de estudos abordaram uma temática completamente nova e extremamente debatida na atualidade, sendo propulsores para a produção de novos conhecimentos compartilhados durante os encontros ou nas apresentações de trabalhos em eventos científicos online. Além disso, os estudantes ampliaram o contato com plataformas digitais que anteriormente não faziam parte do seu cotidiano de forma recorrente. 
SOARES, Tárcila Lorrane Fernandes de Souza; SANTANA; Ícaro Silva de; COMPER, Maria Luiza Caires. Ensino remoto na pandemia de COVID-19: lições aprendidas em um projeto de extensão universitário

A criação de possibilidades para a condução do ensino remoto permitiu a emancipação para novas formas de aprender e ensinar que puderam ser incorporados aos novos ciclos de estudo do projeto de extensão e aos componentes curriculares de semiologia e propedêutica clínica. Em síntese, consideramos que o planejamento compartilhado do plano de aula, o uso do ambiente virtual de aprendizagem como apoio ao ensino remoto, o uso de metodologias ativas como estratégia pedagógica e o gerenciamento da participação dos estudantes são estratégias potencializadoras do ensino remoto por oportunizar a autonomia do estudante no processo de aprendizagem.

Cabe destacar que as estratégias de ensino e aprendizagem aqui sugeridas decorrem da observações e reflexões dos autores diante da experiência do ensino remoto adaptado para dar continuidade aos encontros de ensino do NESPc. Por isso, não existe qualquer pretensão de generalização dos achados. Ao contrário, o interesse é construir novos conhecimentos com base nestas experiências, sobretudo para superar os desafios impostos por esta nova realidade pandêmica.

\section{Referências}

ALMEIDA, Brenda Santana; DE SANTANA SANTOS, Carolyne; COMPER, Maria Luiza Caires. Núcleo de Estudos Em Semiologia e Propedêutica Clínica: Um caminho complementar para a formação em saúde. REVISE-Revista Integrativa em Inovações Tecnológicas nas Ciências da Saúde, v. 4, n. 00, 2019. Disponível

em: $<$ https://www3.ufrb.edu.br/seer/index.php/revise/article/view/1410>. Acesso em: 15 jul 2020 .

ALVES, Lynn. Educação remota: entre a ilusão e a realidade. Interfaces Científicas-Educação, v. 8, n. 3, p. 348-365, 2020. Disponível

em: <https://periodicos.set.edu.br/educacao/article/view/9251>. Acesso em 15 jul 2020.

AQUINO, Estela ML et al. Medidas de distanciamento social no controle da pandemia de COVID-19: potenciais impactos e desafios no Brasil. Ciência \& Saúde Coletiva, v. 25, p. 2423-2446, 2020. Disponível em:<https://www.scielosp.org/article/csc/2020.v25suppl1/2423-2446/pt/>. Acesso em 15 jul 2020

BARBOSA, Andre Machado; VIEGAS, Marco Antônio Serra; BATISTA, Regina Lucia Napolitano Felício Felix. AULAS PRESENCLAIS EM TEMPOS DE PANDEMLA: relatos de experiências de professores do nivel superior sobre as aulas remotas. Revista Augustus, v. 25, n. 51, p. 255280, 2020. Disponível em:

$<$ https://revistas.unisuam.edu.br/index.php/revistaaugustus/article/view/565>. Acesso em: 20 jul 2020.

BERBEL, Neusi Aparecida Navas. As metodologias ativas e a promoção da autonomia de estudantes.

Semina: Ciências Sociais e Humanas, v. 32, n. 1, p. 25-40, 2011. Disponível em: 


\section{Dialogia}

SOARES, Tárcila Lorrane Fernandes de Souza; SANTANA; Ícaro Silva de; COMPER, Maria Luiza Caires. Ensino remoto na pandemia de COVID-19: lições aprendidas em um projeto de extensão universitário

<http://www.uel.br/revistas/uel/index.php/seminasoc/article/view/10326/10999>. Acesso em: 30 set 2020.

BRASIL. Ministério da Educação. CNE. Proposta de parecer sobre reorganização dos calendários escolares e realização de atividades pedagógicas não presenciais durante o periodo de pandemia da COVID-19. Brasília, 2020. Disponível em: https://www.cnm.org.br/cms/images/stories/Links/Texto_Referencia_Reorganizacao_dos_Calendarios_Escolares_-_Pandemia_da_COVID-19_1.pdf. Acesso em: 22 jul 2020.

BRASIL. Ministério da Educação. Portaria $n^{\circ} 544$, de 16 de junho de 2020. Diário Oficial da União, 2020. Disponível em: https://www.in.gov.br/en/web/dou/-/portaria-n-544-de-16-de-junho-de2020-261924872. Acesso em: 22 jul 2020.

CARVALHO, Vitor Oliveira; CONCEIÇÃO, Lino Sergio Rocha; GOIS JR, Miburge Bolivar. COV ID-19 pandemic: Beyond medical education in Brazil. Journal of Cardiac Surgery, v. 35, n. 6, p. 1170, 2020. Disponível em:<https://www.ncbi.nlm.nih.gov/pmc/articles/PMC7307123/>. Acesso em 27 set 2020.

COUTO, Edvaldo Souza; COUTO, Edilece Souza; CRUZ, Ingrid de Magalhães Porto. \# FIQUEEMCASA: EDUCAÇAO NA PANDEMLA DA COVID-19. Interfaces CientíficasEducação, v. 8, n. 3, p. 200-217, 2020. Disponível em:<https://periodicos.set.edu.br/educacao/article/view/8777>. Acesso em 15 jul 2020.

FREIRE, Paulo. Pedagogia da autonomia: saberes necessários à prática educativa. São Paulo: Paz e Terra, 1996.

HERMOGENES, Lucas Ramon et al. A IMPORTÂNCLA DAS DIGITAL SKILLS EM TEMPOS DE CRISE: alguns aplicativos utilizados durante o isolamento social devido à pandemia do covid19. Revista Augustus, v. 25, n. 51, p. 198-218, 2020. Disponível

em: $<$ https://revistas.unisuam.edu.br/index.php/revistaaugustus/article/view/540>. Acesso em 18 jul 2020.

HODGES, Charles et al. As diferenças entre o aprendizado online e o ensino remoto de emergência. Revista da Escola, Professor, Educação e Tecnologia, v. 2, 2020. Disponível em:<http://escribo.com/revista/index.php/escola/article/view/17>. Acesso em 29 jul 2020.

LACERDA, Andreson Lopes de; SILVA, Tatiana da. Materiais e estratégias didáticas em ambiente virtual de Aprendizagem. Rev. Brasileira de Estudos Pedagógicos., Brasília , v. 96, n. 243, p. 321 342, Ago. 2015. Disponível em:

$<$ http://www.scielo.br/scielo.php?script=sci_arttext\&pid=S217666812015000200321\&lng=en\&nrm=iso $>$. Acesso em 30 set 2020. http://dx.doi.org/10.1590/S2176-6681/337812844.

LACERDA, Flávia Cristina Barbosa; SANTOS, Letícia Machado dos. Integralidade na formação do ensino superior: metodologias ativas de aprendizagem. Avaliação (Campinas), Sorocaba, v. 23, n. 3, p. 611-627, Dez. 2018. Disponível em:

$<$ http://www.scielo.br/scielo.php?script=sci_arttext\&pid=S1414-

40772018000300611\&lng=en\&nrm $=$ iso $>$. Acesso

em 23 Set. 2020. http://dx.doi.org/10.1590/s1414-40772018000300003. 
SOARES, Tárcila Lorrane Fernandes de Souza; SANTANA; Ícaro Silva de; COMPER, Maria Luiza Caires. Ensino remoto na pandemia de COVID-19: lições aprendidas em um projeto de extensão universitário

LEÃO, Juliana Alves. As Ferramentas de Interação do Ambiente Virtual de Aprendizagem: Instrumentos que Viabilizam as Inter-Relaçoes entre Professores e Alunos. Revista Gestão Universitária, v. 16, n. 3, 2015. Disponível em:<http://gestaouniversitaria.com.br/artigos/as-ferramentas-de-interacaodo-ambiente-virtual-de-aprendizagem-instrumentos-que-viabilizam-as-inter-relacoes-entreprofessores-e-alunos $>$. Acesso em 23 set 2020.

MARKEL, Julia M.; GUO, Philip J. Designing the Future of Experiential Learning Environments for a Post-COVID World: A Preliminary Case Study. 2020. Disponível em:

$<$ https://www.microsoft.com/en-us/research/uploads/prod/2020/07/NFW-Markel-

Guo.pdf>. Acesso em 30 set 2020.

MOREIRA, J. António; HENRIQUES, Susana; BARROS, Daniela Melaré Vieira. Transitando de um ensino remoto emergencial para uma educação digital em rede, em tempos de pandemia. Dialogia, p. 351364, 2020. Disponível em:<https://repositorioaberto.uab.pt/handle/10400.2/9756>. Acesso em 23 set 2020.

PAIVA, Marlla Rúbya Ferreira et al. Metodologias ativas de ensino-aprendiragem: revisão integrativa.

SANARE-Revista de Políticas Públicas, v. 15, n. 2, 2016. Disponível em:

<https://sanare.emnuvens.com.br/sanare/article/view/1049>. Acesso em 30 set 2020.

PAIVA, Vera Menezes de O. Ambientes virtuais de aprendizagem: implicações epistemológicas. Educação em Revista, v. 26, n. 3, p. 353-370, 2010. Disponível em:

$<$ https://www.scielo.br/scielo.php?pid=S0102-

46982010000300018\&script=sci_arttext\&tlng=pt>. Acesso em: 17 ago 2020.

PRADO, Maria Elisabete. Educação a distância: os ambientes virtuais e algumas possibilidades pedagógicas. Salto para o Futuro/SEED/MEC. Série: Tecnologias e educação: novos tempos, outros rumos. Brasília, p. 20-25, 2001. Disponível

em: $<$ http://www.eadconsultoria.com.br/matapoio/biblioteca/textos_pdf/texto21.pdf $>$. Acesso em: 20 set 2020.

SALVADOR, Pétala Tuani Candido de Oliveira et al . Objeto e ambiente virtual de aprendizagem: análise de conceito. Rev. Bras. Enferm., Brasília, v. 70, n. 3, p. 572-579, June 2017 . Available from <http://www.scielo.br/scielo.php? script=sci_arttext\&pid=S0034-

$71672017000300572 \& \operatorname{lng}=$ en\&nrm $=$ iso $>$. Accesso em 30 set 2020. http://dx.doi.org/10.1590/0034-7167-2016-0123.

SANDHU, Preeti; DE WOLF, Maisie. The impact of COVID-19 on the undergraduate medical curriculum. Medical Education Online, v. 25, n. 1, p. 1764740, 2020. Disponível em:<https://www.tandfonline.com/doi/full/10.1080/10872981.2020.1764740>. Acesso em: 15 jul 2020.

TAKAHASHI, Eduardo Kojy; CARDOSO, Dayane Carvalho. Experimentação remota em atividades de ensino formal: um estudo a partir de periódicos Qualis A. Revista Brasileira de Pesquisa em

Educação em Ciências, v. 11, n. 3, p. 185-208, 2011. Disponível

em: <https://periodicos.ufmg.br/index.php/rbpec/article/view/4214>. Acesso em 17 jul 2020.

VASCONCELLOS, Celso dos Santos. Planejamento: projeto de ensino- aprendiragem e projeto político pedagógico. 21. ed. São Paulo: Libertad, 2010. 205 p. 


\section{Dialogia}

SOARES, Tárcila Lorrane Fernandes de Souza; SANTANA; Ícaro Silva de; COMPER, Maria Luiza Caires. Ensino remoto na pandemia de COVID-19: lições aprendidas em um projeto de extensão universitário

WORLD HEALTH ORGANIZATION et al. Considerations for quarantine of individuals in the context of containment for coronavirus disease (COVID-19) Interim guidance, 29 February 2020. World Health Organization, 2020.

Recebido em: 07 out. 2020 / Aprovado em: 18 nov. 2020

\section{Cite como}

(ABNT NBR 6023:2018)

SOARES, Tárcila Lorrane Fernandes de Souza; SANTANA; Ícaro Silva de; COMPER, Maria Luiza Caires. Ensino remoto na pandemia de COVID-19: lições aprendidas em um projeto de extensão universitário. Dialogia, São Paulo, n. 36, p. 35-48, set./set. 2020. Disponível em: https://doi.org/10.5585/dialogia.n36.18396.

\section{American Psychological Association (APA)}

Soares, T. L. F. S., Santana, I. S, de, \& Comper, M. L. C. (2020, set./dez.). Ensino remoto na pandemia de COVID-19: lições aprendidas em um projeto de extensão universitário. Dialogia, São Paulo, 36, p. 35-48. https:// doi.org/10.5585/dialogia.n36.18396. 